\title{
IMPROVED RADIO STUDIES OF SPACE BY USING NEW EM DEGREES OF FREEDOM
}

\author{
B. Thidé*
}

\begin{abstract}
The use of angular momentum and other EM degrees of freedom provide new ways to improve the diagnostic of radio sources. We show how these methods can be used to extract more information from planetary, solar and heliospheric radio emissions. Examples include imaging of plasma vorticity and sub-Rayleigh resolution by up to one order of magnitude.
\end{abstract}

* Swedish Institute of Space Physics, SE-751 21 Uppsala, Sweden 
